\title{
The Motion of An Inv Nodal Cilium: a Realistic Model Revealing Dynein- Driven Ciliary Motion with Microtubule Mislocalization
}

\author{
Yanan $\mathrm{Yu}^{\mathrm{a}}$ Kyosuke Shinohara ${ }^{\mathrm{b}}$ Huanming $\mathrm{Xu}^{\mathrm{a}}$ Zhenfeng $\mathrm{Li}^{\mathrm{a}}$ \\ Tomoki Nishidac Hiroshi Hamada ${ }^{\mathrm{d}}$ Yuanqing $\mathrm{Xu}^{\mathrm{a}}$ Jingqi Zhou ${ }^{\mathrm{e}}$ \\ Daisy Shao ${ }^{f}$ Xiangchen Lig Duanduan Chen ${ }^{a}$
}

aSchool of Life Science, Beijing Institute of Technology, Beijing, China, bepartment of Biotechnology and Life Science, Tokyo University of Agriculture \& Technology, Tokyo, cJapan Textile Products Quality and Technology Centre, Kobe, 'Laboratory for Organismal Patterning, RIKEN Centre for Developmental Biology, Kobe, Japan, eChongqing Nankai Secondary School, Chongqing, China, ${ }^{\mathrm{f}} \mathrm{Hyde}$ Park Baptist High School, USA, 9Beijing the Eighth High School, Beijing, China

\section{Key Words}

Inv mutant nodal cilia - Dynein activation - Computational simulation • Ultra-high voltage electron tomography

\begin{abstract}
Background/Aims: Nodal cilia that rotate in the ventral node play an important role in establishing left-right asymmetry during embryogenesis; however, inv mutant cilia present abnormal movement and induce laterality defects. The mechanism of their motility, which is regulated by dynein activation and microtubule arrangement, has not been fully understood. This study analyzed the dynein-triggered ciliary motion in the abnormal ultrastructure of the inv mutant, aiming to quantitatively evaluate the influence of microtubule mislocalization on the movement of the cilium. Methods: We established a realistic 3-D model of an inv mutant cilium with an ultrastructure based on tomographic datasets generated by ultra-high voltage electron microscopy. The time-variant activation of the axonemal dynein force was simulated by pairs of point loads and embedded at dynein-mounted positions between adjacent microtubule doublets in this mathematical model. Utilizing the finite element method and deformable grid, the motility of the mutant cilium that is induced by various dynein activation hypotheses was investigated and compared to experimental observation. Results: The results indicate that for the inv mutant, simulations of the ciliary movement with the engagement of dyneins based on the distance-controlled pattern in the partially activation scenario are broadly consistent with the observation; the shortening of the microtubules induces smaller
\end{abstract}

Y. Yu and K. Shinohara contributed equally to this work.

Duanduan Chen

KARGER
School of Life Science, Beijing Institute of Technology Beijing, 100081 (China)

Tel. +86 10 68912154, E-Mail duanduan@bit.edu.cn 
movement amplitudes, while the angles of the mislocalized microtubules affect the pattern of the ciliary movement, and during the ciliary movement, the microtubules swing and twist in the mutant ciliary body. Conclusion: More generally, this study implies that dynein engagement is sensitive to subtle geometric changes in the axoneme, and thus, this geometry greatly influences the integrity of a well-formed ciliary rotation.

(C) 2018 The Author(s)

Published by S. Karger AG, Basel

\section{Introduction}

Cilia are small microtubule-based cellular appendages that provide sensory and motility functions to the cells or the surrounding fluidic environment $[1,2]$, and their dysfunction may induce multiple human genetic disorders [3]. Recent studies found that cilia in the ventral node of the embryo, with a " $9+0$ " ultrastructure, present rotational movement, generate uni-directional flow and thus trigger the establishment of left-right differentiation during embryogenesis [4-10]. In contrast, mutant nodal cilia with abnormal movement fail to produce the leftward flow and thus induce laterality defects [11]. The mechanism of mutant ciliary motility, which is regulated by axonemal dyneins and microtubule arrangement, remains largely unknown.

Previous theories regarding the dynein-driven motility of cilia mainly include the improved geometric clutch theory $[12,13]$, the switching point models $[14,15]$, and the curvature control models [16], which target the beating pattern of " $9+2$ " cilia/flagella. Hilfinger and Jülicher proposed a 3-D description of ciliary dynamics based on dynein motors and microtubule self-organization [17], and proposed a 3-D computer model of the motive force that is controlled by the sliding velocity [18]. For embryonic cilia with a " $9+0$ " structure, it has also been reported that the distance between adjacent doublets in nine doublet microtubules (doublet MTs) changes during mutual sliding. This change in distance may be the mechanism of transmission of dynein activation in the doublet MTs [12, $19,20]$. These studies provide important contributions to our understanding of the ciliary motility as well as cilia-induced fluid motion [21-28]. However, most of the current studies are based on wild-type cilia, and investigations of the ultrastructure and motility of mutant cilia, especially mutant nodal cilia, are still lacking.

The inversin (inv) is a protein produced by a gene that contains 15 tandem repeats of the ankyrin motif and plays a key role in left-right determination during mammalian embryonic development $[29,30]$. Abnormal movement of nodal cilia was observed in the inv mutant mouse embryo [25]; thus, the inv mutation provides a new model for studies on the mechanism of ciliary movement. In our previous studies, the distance-controlled hypothesis for dynein activation between adjacent doublet MTs was proposed for wild-type embryonic cilia. This suggested that the smaller interdoublet distance facilitates the formation of dynein bridges, while the dissociation of dynein bridges occurs with increasing distance [31]. In the current study, we established a 3-D model of an inv mutant embryonic cilium based on ultra-high voltage electron tomography and employed the finite element method to calculate dynein-induced mutant ciliary movement. The internal mechanism of the mutant ciliary motility that is simulated by different modes of dynein activation can thus be investigated, and the influence of the axonemal structure on the ciliary movement can be evaluated.

\section{Materials and Methods}

\section{Imaging Data Acquisition}

This study was approved by the local institutional animal care and use committee (FBS-12-019). ICR mice were obtained from CLEA Japan. Mouse embryos were dissected into phenol red-free Dulbecco's Modified Eagle's medium (DMEM) supplemented with $10 \%$ fetal bovine serum (Invitrogen). The developmental stage (from early head fold to two-somite) of the embryos was determined on the basis of morphology and the number of somites. Dissected embryos were cultured at $37^{\circ} \mathrm{C}$ in phenol red-free DMEM 


\section{Cellular Physiology Cell Physiol Biochem 2018;51:2843-2857

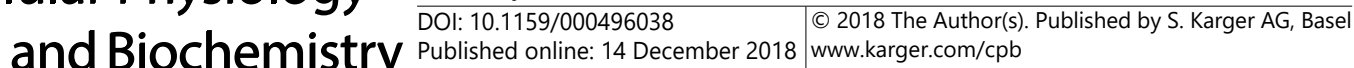 \\ Yu et al.: Motility of Inv Mutant Nodal Cilia}

supplemented with 75\% rat serum (Invitrogen). The experimental data obtained in our previous study [32] were analyzed in the previous and the present studies, which focused on the Taxol-treated nodal cilia and inv mutant cilium, respectively.

To obtain images of the inv nodal cilium, a tomographic dataset with $1.6 \mathrm{~nm}$ slice thickness was obtained via an ultra-high-voltage electron microscope (H-3000, Hitachi) operating at $2 \mathrm{MeV}$ (Fig. 1a). The sections of the sample were set onto Formvar-coated 75- mesh molybdenum grids. To construct tomograms, gold nanoparticles $(20 \mathrm{~nm})$ were deposited on both surfaces. Furthermore, evaporation coating of carbon was carried out to prevent charging of sections. Images were captured using an F415S slow-scan CCD camera (4096×4096 pixels). Both 3-D reconstruction and modelling were performed with the IMOD imageprocessing package [33].

To characterize the dynamic properties of inv ciliary rotation, a high-speed camera and a point-tracking system were applied. The node cavity was filled with phenol red-free DMEM supplemented with $10 \%$ fetal bovine serum, and rotation of the node cilium was observed at the apical cell surface of the node cavity for $2 \mathrm{~s}$ (100 frames/s) with the use of a high-speed CMOS camera (HAS-500M, Detect). The camera was connected to an Axiovert2 microscope equipped with a 100× oil-immersion objective lens for observation of individual node sections. The specimen was observed with transmitted light from a halogen lamp. Timeseries images were captured at a resolution of 1024 by 992 pixels, with a pixel resolution of 0.082 by 0.082 $\mu \mathrm{m}$. The recorded video was converted to a series of image frames. The ciliary tip was segmented, and the centroid of this region of interest was tracked to obtain the ciliary tip trajectory.

\section{Establishment of the Realistic 3-D Ciliary Model}

The image datasets were processed and segmented (Fig. 1b) in an open-source modelling software suite (IMOD 4.5), to extract the geometric data of each doublet MT and the shape of the ciliary surface (Fig. 1c). These morphological data were then imported into a mesh generator (CFD-GEOM, ESI Group) to reconstruct the 3-D ultrastructure of the mutant embryonic cilium and to establish the volume mesh (Fig. 1d). To reduce the computational complexity, the cross-section of doublet MTs is simplified into an ellipse with a major radius of $30 \mathrm{~nm}$ and a minor radius of $17 \mathrm{~nm}$ [31]. In contrast to the ultrastructure of wild-type cilium, where the nine doublet MTs surround the center line of the ciliary body, the doublet MTs in the mutant cilium show obvious "mislocalization" and significant variation in length. As shown in Fig. 1c-d, the arrangement of doublet MTs near the ciliary root is organized, while mislocalization of MTs occurs in the middle and upper region of the ciliary body. Thus, the nine doublet MTs in the mutant case have been numbered clockwise based on their arrangement near the ciliary root. The doublet MT-1 has the greatest inclination and increase in length, which extends outward between doublets MT-4 and - 5 and establishes a branch from the main body of the cilium. Other doublet MTs also show mislocalization, and doublets MT-2, -3 and -9 show the most significant shortening in length. To generate the computational model, unstructured tetrahedral elements were used to discretize the ciliary body, while triangular prisms were applied to generate the volume mesh of the doublet MTs. The model of the inv mutant cilium contains $1,238,389$ finite elements in total.

\section{Mechanical Properties}

The Young's modulus of the doublet MTs has been assigned as $8 \times 10^{8} \mathrm{~N} / \mathrm{m}^{2}$ [34-36]. The mechanical properties of the ciliary cytoplasm are difficult to assess; they can hardly be measured in mutant individuals and may vary greatly among species. In our previous study of nodal cilia, a parameter study was conducted on the cytoplasmic elastic modulus in the range of $5-10^{3} \mathrm{~Pa}$ [31]. The parameter study proposed that the elastic modulus of $100 \mathrm{~Pa}$ is suitable for the ciliary cytoplasm in the node [31]. Thus, in the current study, this value is also employed in the computational model.

When the dynein is activated, the dynein motors located on one doublet MT form a transient dynein bridge to the adjacent counterpart and generate counter-directional forces. The force pushes the target doublet MT to move towards the cilia tip [37]. The functions of the activated dyneins are modelled as pairs of point loads that work in opposite directions. The force exerted by a single axoneme dynein arm is reported to be $1-10 \mathrm{pN}$ [38-40]. The intermediate value of $5 \mathrm{pN}$ is used in this study. The pairs of point loads act on corresponding sites located on the dynein-located doublet and the target adjacent doublet. The forces of other deactivated dyneins are set to zero.

\section{KARGER}




\section{Cellular Physiology Cell Physiol Biochem 2018;51:2843-2857

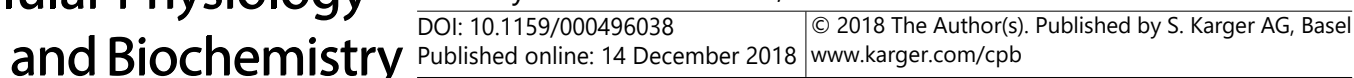 \\ Yu et al.: Motility of Inv Mutant Nodal Cilia}

\section{Numerical Methods}

The finite element method has been applied to numerically solve the complex mathematical models in the complicated geometrical domain of the cilium. In this paper, the entire ciliary domain under investigation can be approximated as an assembly of discrete elements, and the geometry and the displacements of each element are completely described by the geometric positions and displacements of the element nodal points. Dynein forces are embedded on particular element nodes with spatial and temporal arrangements based on various dynein activation hypotheses. Mechanical equations have been solved by a stress solver using CFD-ACE+ (ESI CFD, France). The equilibrium of the system is described as

$$
K D=P,(1)
$$

where $D$ denotes the node displacement vector, $K$ is the stiffness matrix, and $P$ denotes the load vector. The global stiffness matrix $K$ is assembled by the stiffness matrix of each element. During a simulation, the mechanical forces generated by the activated dyneins are simulated by modifying the load vector $P$ with point loads on particular grid nodes.

The components are treated as elastic continuous materials, utilizing solid-body elasticity analogy [41] to achieve grid deformation. The equation governing the displacement of the internal nodes can be written as

$$
\nabla \cdot \sigma+f=0,(2)
$$

where $f$ is the body force and $\sigma$ is the stress tensor. The body force $f$ is zero because of all the displacement results from the specified boundary node displacements. The strain tensor $\varepsilon$ is defined in terms of the displacement vector $u$ :

$$
\varepsilon=1 / 2\left(\nabla u+\nabla u^{T}\right),(3)
$$

The stress tensor $\sigma$ is related to the strain tensor $\varepsilon$ as

$$
\varepsilon=\lambda \operatorname{Tr}(\varepsilon) I+2 \mu \varepsilon,(4)
$$

where $\operatorname{Tr}$ is the trace operator, $I$ is the identity matrix, and $\lambda$ and $\mu$ are the Lamé coefficients, expressed in terms of material properties as

$$
\begin{aligned}
& \mu=E / 2(1+\gamma),(5) \\
& \lambda=\gamma E /[(1+\gamma)(1-2 \gamma)],(6)
\end{aligned}
$$

In Eqs. 5 and 6, $E$ is the modulus of elasticity and $\gamma$ is Poisson's ratio. The governing equation is then rewritten with $u$ as the primitive variable:

$\nabla \cdot\left[\mu \nabla u+(\nabla u)^{T}\right]+\nabla(\lambda \nabla \cdot u)+f=0,(7)$

Consider the elastic body occupying a bounded region $\Omega$ with boundary $\Gamma$. The Dirichlet and Neumanntype boundary conditions for mesh motion are imposed by the motion of boundaries and interfaces, respectively, as

$$
\begin{aligned}
& u=g \text { on } \Gamma_{g^{\prime}} \text { (8) } \\
& n \cdot \sigma=h \text { on } \Gamma_{h^{\prime}} \text { (9) }
\end{aligned}
$$

where $\Gamma_{g}$ and $\Gamma_{h}$ are complementary subsets of $\Gamma$. The finite element function spaces are constructed as

$$
\begin{aligned}
& S^{h}=\left\{u^{h} \mid u^{h} \in\left[H^{h}(\Omega)\right]^{n}, u^{h}=g^{h} \text { on } \Gamma_{g}\right\},(10) \\
& W^{h}=\left\{w^{h} \mid w^{h} \in\left[H^{h}(\Omega)\right]^{n}, w^{h}=0 \text { on } \Gamma_{g}\right\},(11)
\end{aligned}
$$


Where $H^{h}(\Omega)$ is
the finite-dimensional function space over $\Omega$. This space is formed over the element domain using continuous firstorder polynomials in space. During the temporal period, distinct time steps were solved independently, finding $u^{h} \in W^{h}$ such that $\forall w^{h} \in W^{h}$

$\int_{\Omega} \varepsilon\left(w^{h}\right): \sigma\left(u^{h}\right) \mathrm{d} \Omega \quad-\int_{\Omega}$ $w^{h} \cdot \mathrm{fd} \Omega=\int_{\Gamma h} w^{h} \cdot \bar{h} \mathrm{~d} \Gamma,(12)$

The computational platform we used allows the self-induced deformation and movement of the protein structure system to be calculated.

\section{Results}

Morphological Analysis

The ultrastructure of the cilia, especially the morphology of doublet MTs, plays a crucial role in controlling the movement of the cilium [32]. The main ultrastructure of wild-type nodal cilia contains a ring of nine outer doublet MTs, which are approximately evenly distributed around the center. However, mutant nodal cilia often present abnormal structures or mislocalization of the MTs [32]. To understand the mechanism of the abnormal movement of the studied inv mutant cilium, it is necessary to reveal its internal MT mislocalization and establish the relationship between the movement and the morphological

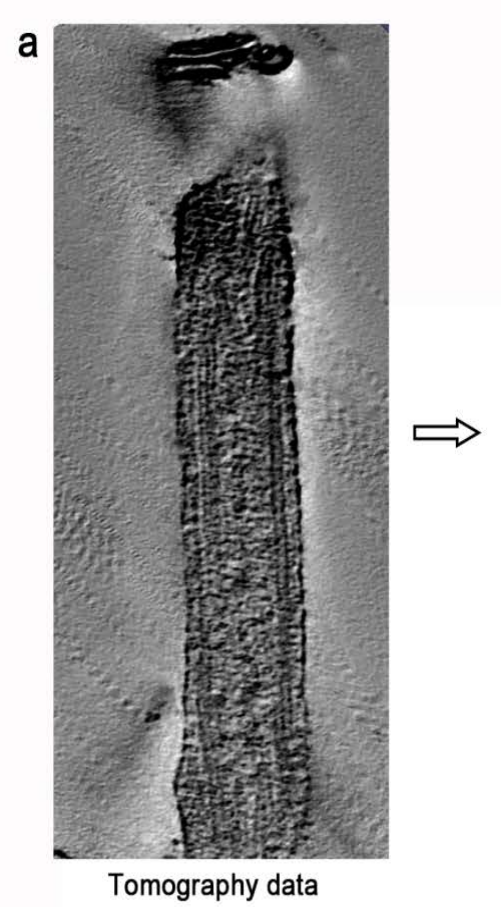

b

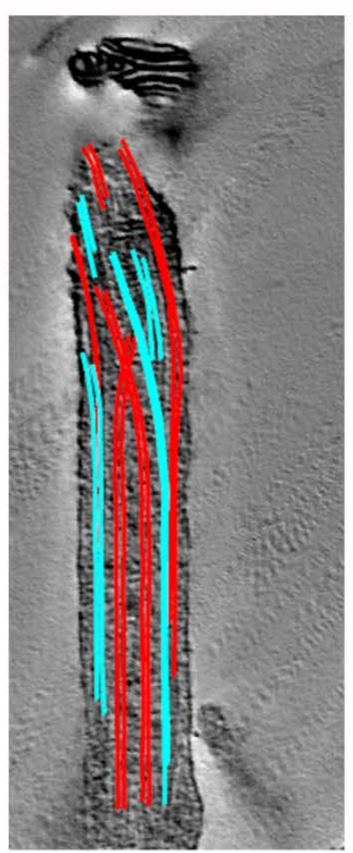

Image segmentation

C
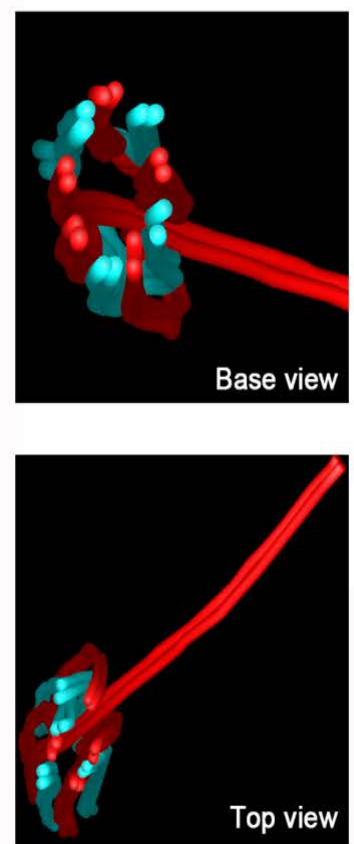

Structure of DMTs

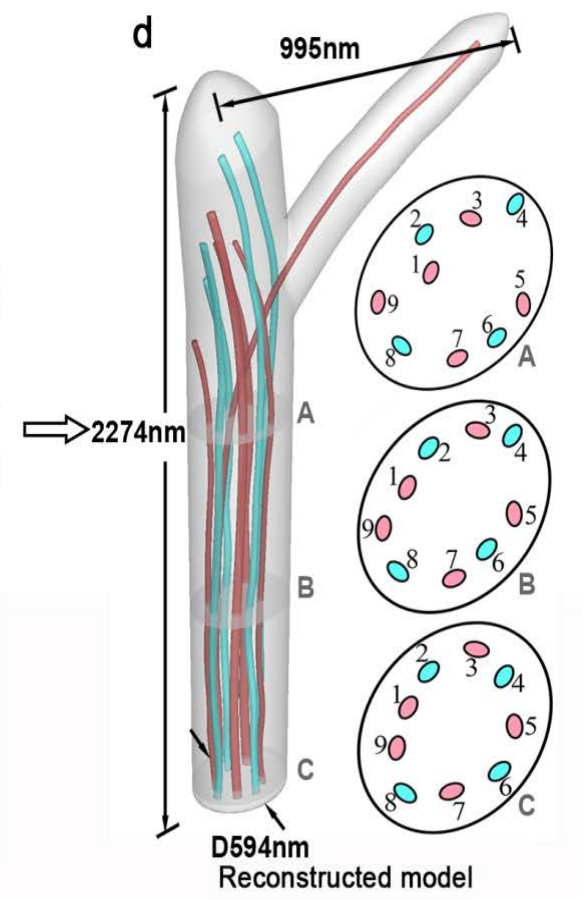

Fig. 1. Three-dimensional ultrastructure of inv mutant cilium and the work flow of model reconstruction. (a) shows the image datasets by ultra-high voltage electron tomography; (b) displays the image segmentation of the doublet microtubules; (c) shows the structure of nine doublet MTs; and (d) shows the reconstructed 3-D model of the mutant cilium. 


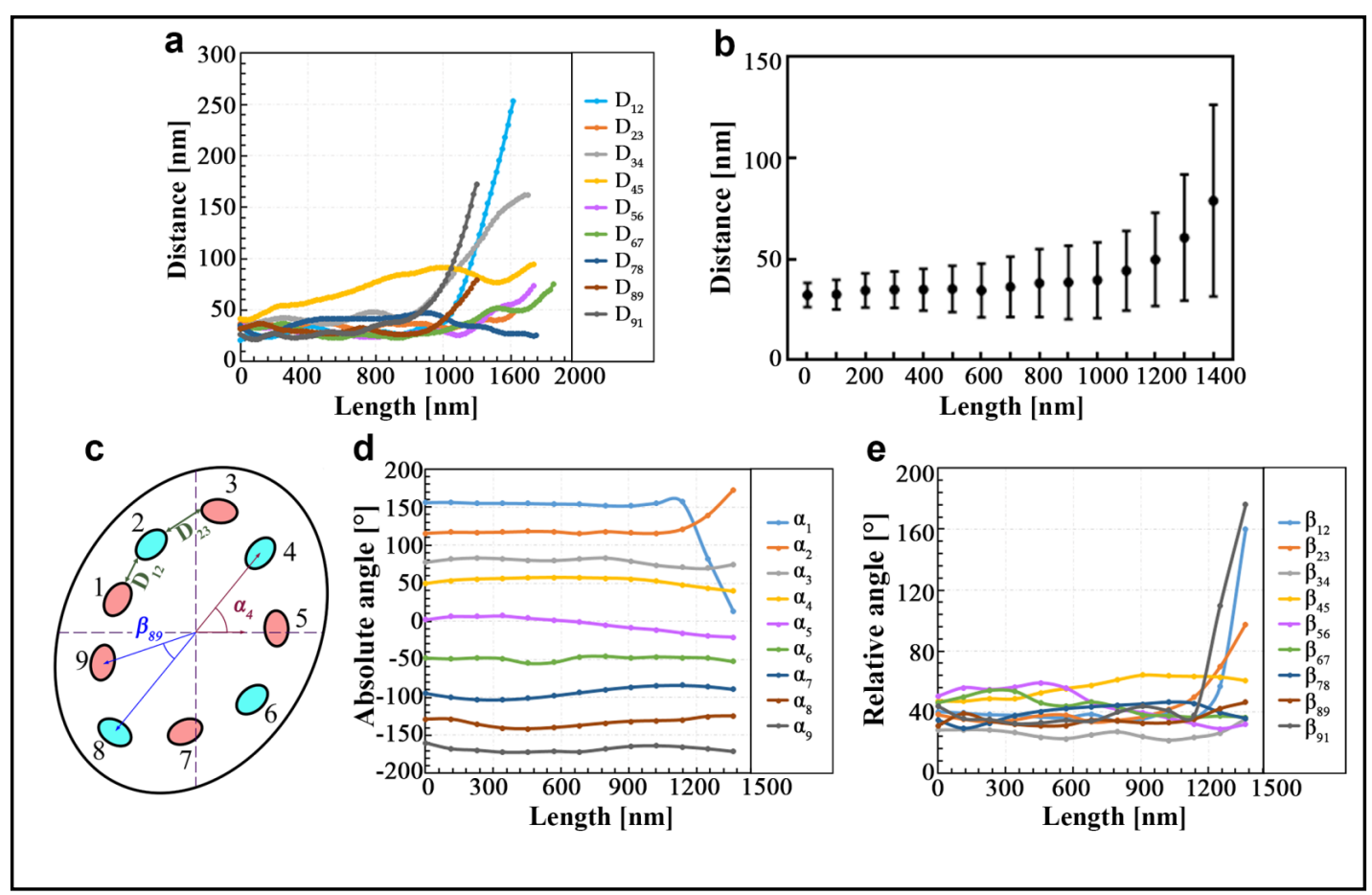

Fig. 2. Morphological analysis of the inv mutant nodal cilia. (a) shows the interdoublet distance along the ciliary length; (b) shows the averaged interdoublet distance; (c) describes the measurements of the angles between adjacent doublets $(\beta)$ and between the microtubule and the horizontal axis ( $\alpha$ ); (d) and (e) displays the two angles of doublet MTs.

features. Thus, in the current study, a realistic model of the inv cilium was reconstructed based on electron tomography.

Fig. 2a presents the distance between adjacent doublet MTs $\left(\mathrm{D}_{m n}\right.$, where $m$ and $n$ denote the numbers of the adjacent doublet MTs) from the base to the tip of the cilium. The interdoublet distance is measured by the distance between adjacent doublet MTs at the corresponding position with equal arclength along the cilium starting from the base. Different from the equal space between adjacent doublet MTs in wild-type cilia, the inv mutant shows a highly complex variation in the distance between doublet MTs. The lower region of the doublet MTs that is close to the base cell is generally organized, while the upper region shows a high degree of abnormality (Fig. 1c). Fig. 2b shows the averaged interdoublet distance along the length of the cilium. In general, the distances between most doublet MTs gradually increase from the ciliary base to the tip, while nonlinear. The standard deviation of distances along the ciliary length between doublets MT-2 and -3 was $3.086 \mathrm{~nm}$, which is the minimum among all of the pairs of doublet MTs, while the maximum is $56.53 \mathrm{~nm}$, occurring between doublets MT- 1 and -2 .

Apart from the distance analysis, twisting of the doublet MTs was also studied. As shown in Fig. 2c, the two angles $(\alpha$ and $\beta$ ) of the cross-section of the cilium along its length were measured. With the centroid of the cross-section of the cilium assigned as the origin, $\alpha_{n}$, where $n$ indicates the doublet MT number, denotes the angle between each doublet MT and the horizontal direction; while $\beta_{m n}$, where $m$ and $n$ indicate the doublet MT numbers, denotes the angle between each pair of adjacent doublet MTs. Fig. $2 \mathrm{~d}$ and Fig. $2 \mathrm{e}$ show $\alpha$ and $\beta$, respectively. The value of $\alpha$ along the length of the cilium (Fig. 2d) implies that, different from wild-type doublet MTs, mutant doublet MTs are not perpendicular to the cross-section of the ciliary body. Moreover, comparing Fig. 2e to Fig. 2a, suggests that the variations of interdoublet distances are not exactly consistent with the change in the angle between doublet MTs, indicating the twisting feature of the doublet MTs in the inv cilium. 


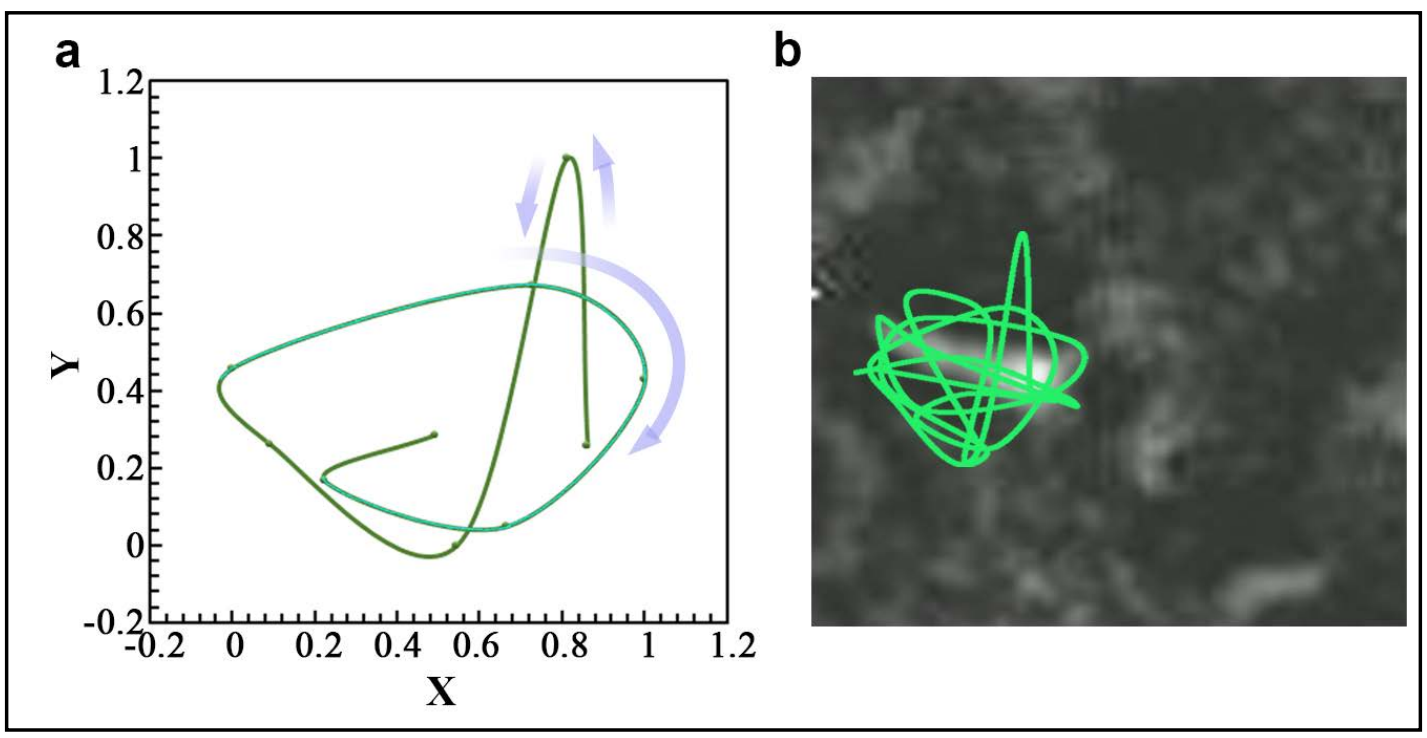

Fig. 3. (a) shows the extracted ciliary tip trajectory of the inv mutant cilium; and (b) shows one snapshot of the observation video.

\section{The Movement Pattern of the Mutant Nodal Cilium}

The rotational cycle of nodal cilia is reported to be approximately $0.1 \mathrm{~s}$ [42]. In the recorded video of the inv cilium, a similar movement cycle was measured. By extracting the tip trajectory of the inv cilium, motility that combines rotation and beating was found, while in wild-type cilia, the movement is a nearly circular rotation. The tip amplitude of the rotation of the wild-type cilium is approximately $64 \%$ of the ciliary length (converted based on the apex angle of the conic rotation of normal cilia [25]), while the maximum amplitude for the mutant cilium is up to $51.9 \%$ of the ciliary length, and the average amplitude is $22.8 \%$ of the ciliary length. Fig. 3a shows the extracted trajectory of an inv cilium. Fig. 3b shows one snapshot of the recorded video, and Video S1 (For all supplemental material see www. karger.com/ 10.1159/000496038/) shows its complete movement.

The movement of the inv mutant cilium is directly related to downstream embryogenesis [43]. Changes in the structural arrangement of doublet MTs in the axoneme strongly affect the regulation of ciliary movement in the mutant cilia; however, so far, the mechanism relating the structure and arrangement of MTs and the motility of the entire cilium has not been fully understood. Thus, based on the 3-D reconstructed model, the motility of the studied mutant cilium is simulated by finite element analysis to investigate the ciliary response of the dynein forces based on the abnormal doublet MT structures. Similar to our previous study [31], the binding of dynein bridges was assumed to occur from the ciliary base to the tip along each individual doublet; dissociation, on the contrary, and the transference sequence of dynein activation is clockwise among the nine doublets. In our previous study on wild-type nodal cilia, we compared ciliary motility based on various dynein activation patterns along the ciliary length. The dynein attachment and detachment were controlled by linear, sinusoid, circular or elliptical functions, and the sinusoidal function was able to produce circular rotation of the ciliary movement in the computational tests. Thus, in the current study, the sinusoid activation mode is applied as the dynein activation and dissociation pattern. In other words, the attachment/detachment occurs more rapidly near the base of the cilia and more slowly when it is close to the ciliary tip. The function [31] of the sinusoid activation mode is shown as

$$
h_{\text {sine }}=h_{n} \cdot \sin \left(k_{s} \cdot t\right) \text {, }
$$


where $t$ denotes time with the unit of seconds, $h$ denotes the dynein activation height with the unit of micrometers, and $n$ indicates the doublet MT number. The parameters of Eq.13 are displayed in Table 1.

In the studied case, we consider the cilium to be at an unloaded equilibrium in the base model. The dynein arms in the inv mutant are reported to be intact [44]; however, due to the variant distance between the doublet MTs, it is different to determine whether the dyneins can effectively reach the target doublet MTs. Thus, in the current study, two scenarios of dynein activation are proposed: the fully activated scenario considers that all of the dyneins along the doublet MTs can be effectively functioned, while the partially activated scenario suggests that the dyneins can only be functional in particular regions due to the distance between doublet MTs. In silico studies were conducted for both scenarios, and the simulated results were compared to the video-recorded mutant ciliary motion to evaluate the possible mechanical mechanism of the ciliary motility of inv mutants.

\section{Fully activated scenario}

In this scenario, all of the dyneins along each set of doublet MTs are assumed to be activated in the cilia. Two types of time control patterns have been considered: timeindependent and time-lapped dynein activations. In the time-independent pattern, the dynein binding in doublets $n+1$ and $n+2$ initiates exactly after the dynein attachment finishes between doublets $n$ and $n+1$, while the time-lapped pattern means the dynein activation in doublets $n+1$ and $n+2$ can be initiated before the dynein binding is completed in doublets $n$ and $n+1$. As discussed in our previous study [31], the time-lapped pattern is more likely to establish the circular rotation of the nodal cilia.

Fig. 4a displays the calculated results of the third periodic tip trajectories of the dyneindriven ciliary motion under the time-independent and time-lapped patterns. In the timelapped pattern, the overlapped time is defined by $\mathrm{T}_{\mathrm{L}}=\mathrm{k} \cdot \mathrm{T}_{\mathrm{MT}}$, where, $\mathrm{k}=0.6$ and $\mathrm{T}_{\mathrm{MT}}$ is the completion time of the dynein activity along individual doublet MTs, as proposed in our previous study [31]. Although the general trajectory is irregular, both patterns result in a combination of clockwise rotation and planar beating of the cilium. Fig. $4 \mathrm{~b}$ displays the ciliary tip displacement in these two patterns. By comparing Fig. 4a with Fig. 3a, general rotation and beating patterns can be captured in both of the simulated and observed results; however, the details of the movement present differences.

Table 1. Parameter list

\begin{tabular}{lccccc}
\hline Parameter (unit) & Value & Parameter(unit) & Value & Parameter(unit) & Value \\
\hline $\mathrm{h}_{1}(\mu \mathrm{m})$ & 1.65 & $\mathrm{~h}_{5}(\mu \mathrm{m})$ & 1.725 & $\mathrm{~h}_{9}(\mu \mathrm{m})$ & 1.4 \\
$\mathrm{~h}_{2}(\mu \mathrm{m})$ & 1.62 & $\mathrm{~h}_{6}(\mu \mathrm{m})$ & 2.06 & $\mathrm{ks}_{5}(\mathrm{rad} / \mathrm{s})$ & 157.08 \\
$\mathrm{~h}_{3}(\mu \mathrm{m})$ & 1.71 & $\mathrm{~h}_{7}(\mu \mathrm{m})$ & 1.87 & & \\
$\mathrm{~h}_{4}(\mu \mathrm{m})$ & 2.05 & $\mathrm{~h}_{8}(\mu \mathrm{m})$ & 1.77 & & \\
\hline
\end{tabular}
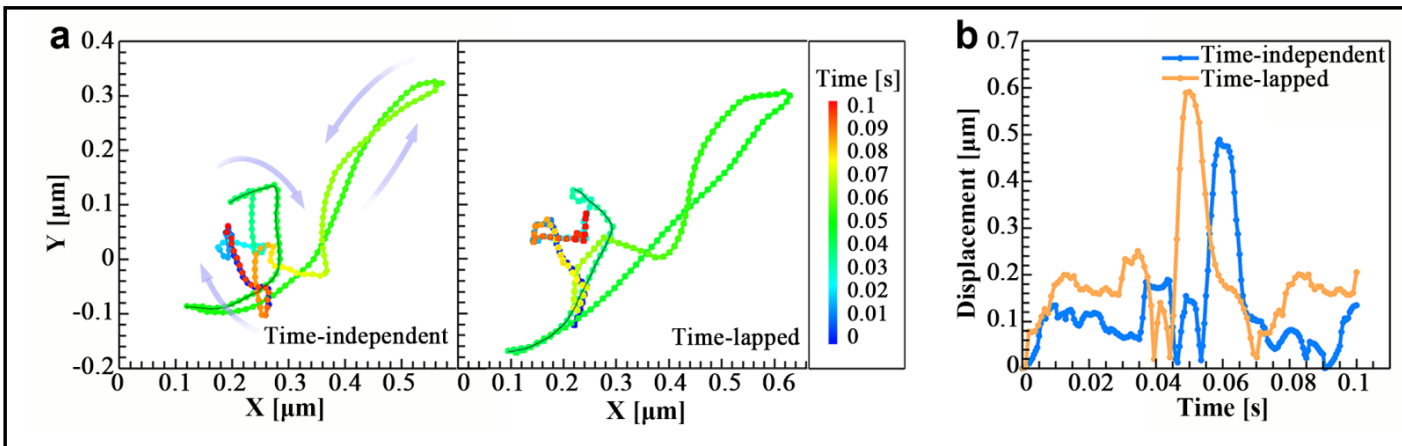

Fig. 4. The simulated motion of the inv cilium under the fully activation scenario. (a) shows the tip trajectory under the time-independent (left) and time-lapped (right) patterns; and (b) shows the displacement difference between the two results. 
The results indicate that in this scenario, the timing patterns have little effect on the general ciliary movement (Fig. 4a) or the movement magnitude (Fig. 4b). In our study on wildtype cilia with uniform morphology of doublet MTs, the timing patterns play an important role [31]. This implies that in the mutant cilium with abnormal arrangement of the doublet MTs, the doublet MT structure may have a more significant effect on ciliary movement than the activation timing of the dyneins. In this study, because we focus on the structure-induced ciliary movement, only the time-independent pattern of the dynein activation is investigated in the following simulations.

\section{Partially activated scenario}

In this section, different from the fully activated scenario, we assume that only a portion of the dyneins along each doublet MT can be effectively activated. Two patterns are simulated: one assumes that the dynein activation only occurs along $75 \%$ of the doublet MT length (from base to tip), where the averaged interdoublet distance reaches $50 \mathrm{~nm}$ and increases greatly beyond this height (Fig. 2b); the other assumes dynein activation at a specific interdoublet distance, and this critical distance was assigned as $50 \mathrm{~nm}[32,45]$. The left panel of Fig. 5a shows the third periodic result of the pattern with $75 \%$ length of the doublet MTs that possess dynein activation. The overall movement tendency of the cilium (rotation and planar beating) is similar to that in Fig. 4a; however, the ciliary rotation trajectory is closer to the circular arc shown in Fig. 5a, and the ciliary beating magnitude is smaller. As might be anticipated, the number of dyneins that are directly activated affects the amplitude of the movement, which is the same as that found in wild-type cilia; however, it also affects the movement pattern to some degree in the mutant case, which is possibly related to the abnormal structure of the doublet MTs.

To further investigate the distance-dependent pattern, the distance between doublets was calculated. As shown in Fig. 6, the ratio of the interdoublet distance variation to its initial value was analyzed. At the initial state, the cilium is immobilized; thus, the ratio is zero. Fig. 6 a and Fig. 6b show the interdoublet distance variations along the microtubules when dynein attachment between doublets MT-1 and -2 (Fig. 6c) is the half and full ciliary length, respectively. It can be found that when dyneins are partially activated along doublet MT-1, most of the regions along the microtubules present an increased distance between doublets MT-1 and -2. The most increased portion occurred in the middle part of the doublet, the largest change of which accounted for $89.76 \%$ of the initial distance. At the same time, the distance between the doublet pair of MT-2 and - 3 decreases; the maximum change is $-34.27 \%$. When the dynein bridge binds between doublets MT-2 and -3 (Fig. 6f), and the dynein arm detaches between doublets MT- 1 and -2 at the same time, the interdoublet distance variation continues. Fig. $6 \mathrm{~d}$ and Fig. $6 \mathrm{e}$ show the change in distance at the midpoint and at the end of this period, respectively. As shown in Fig. 6e, the distance between doublets MT-2 and -3 shows the largest increase, and the maximum increase distance is equivalent to $56.07 \%$ of
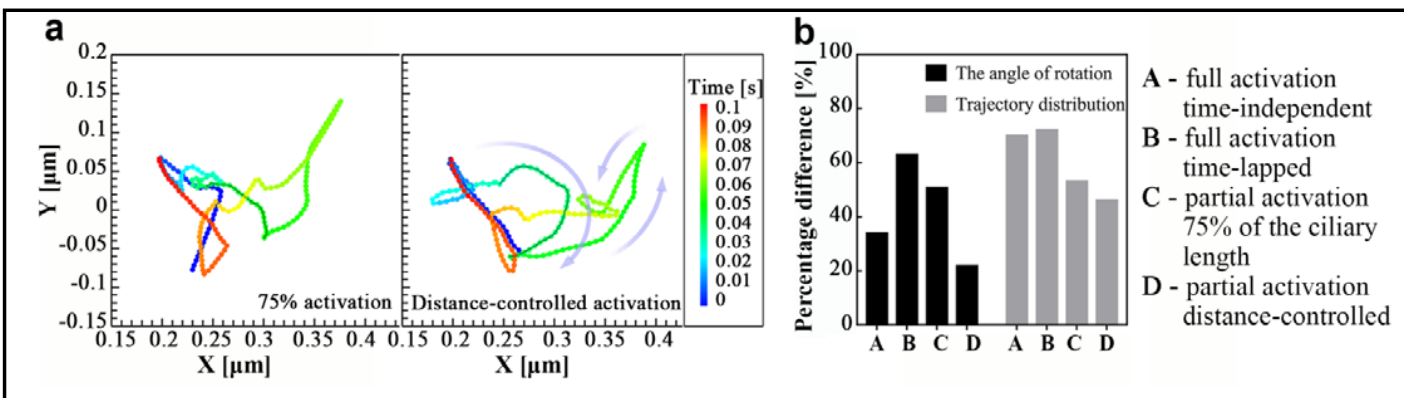

Fig. 5. The simulated motion of the inv cilium under the partially activation scenario. (a) shows the tip trajectory when $75 \%$ of the dyneins along each doublet MT are activated (left) and the results of the distance-controlled model with $50 \mathrm{~nm}$ as the dynein activation distance criteria (right); and (b) displays the quantitative comparison of the ciliary movement pattern to the video-recorded results. 


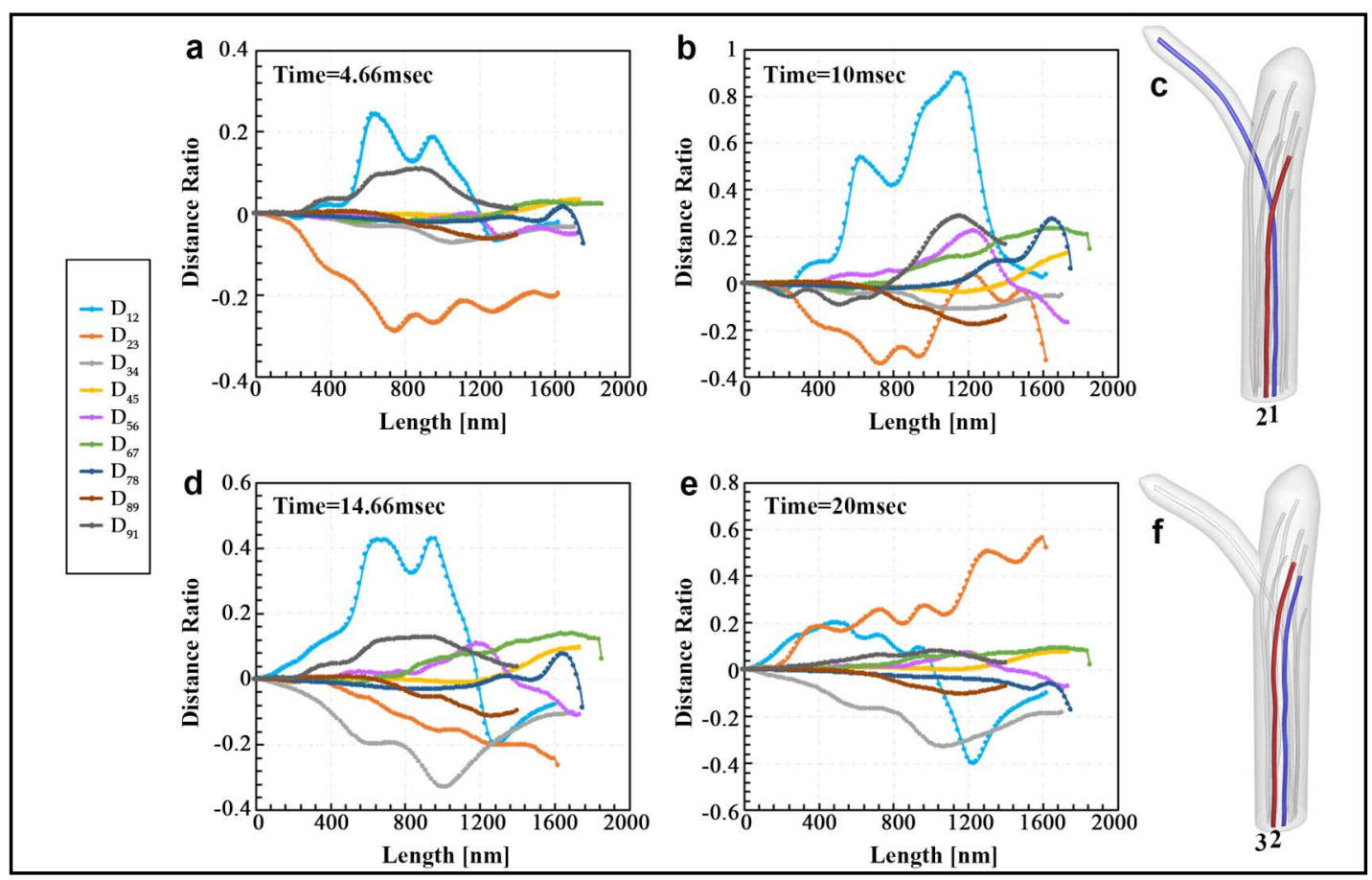

Fig. 6. Variation of the interdoublet distances. (a) and (b) shows the interdoublet distance variation when dynein formation occurs between doublets MT-1 and -2 at different times; (d) and (e) shows the distance variation when dynein formation occurs between doublets MT-2 and -3. (c) and (f) illustrate the dynein formation between doublets MT- 1 and -2 as well as doublets MT- 2 and -3 , respectively.

the initial distance. Meanwhile, the distance between doublets MT-3 and -4 is reduced, and the maximum change is $-40.12 \%$. In addition, the distances between other doublets show different patterns of change.

The results show that for the inv mutant, the distance between adjacent doublet MTs does not exactly match the distance variation pattern for the wild-type counterpart. The change in the interdoublet distance in the partially activation scenario is similar to that obtained under fully activation, suggesting that the dynein activation number of each doublet MT may not be responsible for the difference in the distance variation between the inv mutant and the wild-type cilia. The right panel of Fig. 5a shows the simulated cilia tip trajectory under the $50 \mathrm{~nm}$ interdoublet-distance pattern. To quantitatively compare the similarities of the four simulation results to the observed result, the trajectory pattern and the rotational angle were further studied. The extraction trajectory and the four calculation trajectories are normalized to calculate the rotational angles of the main rotation trajectories. Each trajectory is discretized with equal intervals and divided into four regions by the horizontal and vertical axes of the centroid of the trajectory to calculate the difference of the trajectory distribution between the four computational studies and the observation-extracted data. The percentage difference is defined as $\left(S_{S}-S_{0}\right) / S_{0} \times 100 \%$, where $S_{S}$ denotes the absolute value of the corresponding parameter for the simulated trajectory and $S_{o}$ denotes the observationextracted trajectory. As shown in Fig. 5b, the distance-controlled pattern in the partially activation scenario presents the most similarity to the observed image.

Furthermore, the variations in the angle of each microtubule during ciliary motion are analyzed (Fig. 7). For mutant nodal cilia, each doublet MT swings during the sliding of a pair of doublet microtubules (Fig. 7a), which is significantly different from wild-type cilia (Fig. 7c). Fig. $7 \mathrm{~b}$ and the variation of interdoublet distance also indicate that the doublet MTs of the mutant cilium swing and twist during the ciliary motion. However, for wild-type cilia, the angle variation is consistent. 

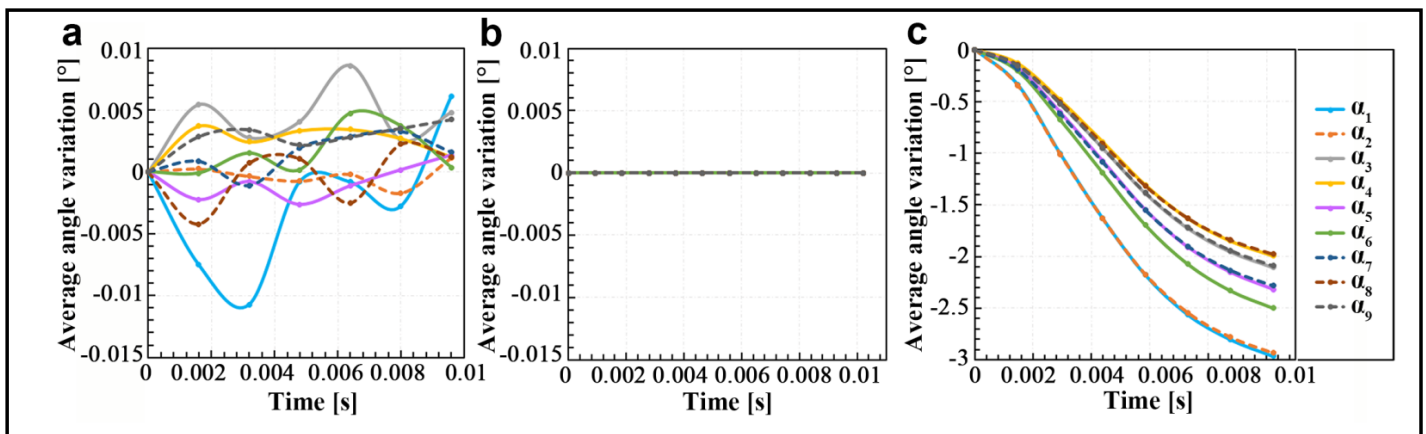

Fig. 7. The angle variation of each doublet MT during the sliding motion between doublets MT-1 and -2 . (a) shows the angle variations of inv mutant nodal cilia when $75 \%$ of the dyneins along each doublet MT are activated; (b) shows the angle variations of the distance-controlled model; and (c) shows the angle variations of the wild-type nodal ciliary mod.

\section{Discussion}

In inv cilia, due to the abnormal arrangement of the doublet MTs along the ciliary length, 3-D reconstructed models cannot be well established based on one or several cross-sectional scans of the ciliary body; therefore, tomographic datasets with a series of fine slices along the ciliary length are needed. In the current study, a model of the ultrastructure of an inv mutant cilium was established based on ultra-high voltage electronic tomography. Consistent with other reports [46], the doublet MTs in this model show turbulent or convoluted structures (Fig. 1c) with no defects in the dynein arms [44], making the suitable for application in testing dynein activation patterns and in analyzing the ciliary motility affected by the doublet MT structures. Ciliary movement was also recorded by a high-speed CMOS camera that was connected to a microscope. By comparing the simulated ciliary motility to the video recorded results, we evaluated the validity of the proposed dynein activation hypothesis and investigated the morphologic influence of the doublet MTs on ciliary motion.

Based on our previous studies, dynein activation is believed to transfer clockwise among nine doublet MTs. Because the dyneins are intact, two scenarios of the dynein activation capability are discussed. In the fully activated scenario, we investigated two time-controlled models: the time-independent pattern and the time-lapped pattern of the dynein activities along doublet MTs. In comparing the tip trajectory of the computed ciliary movement, both patterns show similarities, while the time phase is slightly different (Fig. 3b). Since we focus on the movement pattern of the mutant cilium, the time-independent model is considered adequate to simulate ciliary motion. By comparing its results to the video recorded results, it is found that both the rotation and planar beating pattern can be captured by this model in general; however, the magnitudes of movement are not exactly the same. Thus, the partially activation scenario is further discussed.

In the partially activation scenario, the dynein activation region is first assigned to $75 \%$ of each doublet MT length. The results indicate that the overall ciliary movement by this $75 \%$ partially activation scenario is generally similar to that in the fully activated model; however, the amplitude of movement is smaller, and the movement trajectory of the ciliary tip is closer to a circular arc (Fig. 5a). By comparing this to the video recorded results, it can be found that the partially activation scenario can produce more similar results for the ciliary motility. More importantly, in our previous study on wild-type cilia, we found that partially activated dyneins may result in a smaller bending magnitude of the cilia but do not influence the moving pattern [31]. These results on the mutant cilium indicate that due to the abnormal structure of the doublet MTs, less activated dyneins also affect the moving pattern to some degree. Moreover, in this case, the ciliary rotation angle is smaller than wild-type nodal cilia, which would weaken the nodal flow produced by the ciliary movement. Some previous studies supported this result $[43,46]$. This means that the axonemal morphologic change may affect 
the amount of activated dyneins and thus influence the effective ciliary motility. We assume that this influence on the activated dyneins is due to the interdoublet distance. Thus, the second case studied in this scenario is distance-dependent dynein activation. The critical distance is assigned as $50 \mathrm{~nm}$ [32]; in other words, the distances between doublets may influence the formation of dynein bridges. Detailed distance analysis has been conducted on the partially activation scenario, and the results show that the distance-dependent dynein activation pattern can produce the most similar movement pattern of the inv mutant by comparison to the recorded realistic ciliary movement (Fig. 5b).

To further reveal the mechanism of the ciliary motion due to the abnormal doublet MTs, the variations in the angle (the value of the difference between the instant $\alpha_{m}$ and its initial value) along each doublet MT during ciliary motion were analyzed (Fig. 7). The results show that the difference in angle variation of the size between the inv mutant and the wild-type nodal cilia may be due to the magnitude of the movement and the different geometry. For the inv mutant, each doublet MT swings during the sliding of a pair of doublet MTs, whereas the doublet MTs of wild-type nodal cilia only move in a clockwise direction. This suggests that the MT mislocalization affects the angle of doublet MT, and then affects the interdoublet distance, which therefore induces an abnormal movement of the ciliary body. Compared to the "geometric clutch model" [12, 13, 47], our study shows much difference for the mutant ciliary simulation. The distance between the doublet MTs might be one crucial factor for dynein activation due to the abnormal morphology in the axoneme.

This study simulates the dynein-driven inv mutant ciliary movement based on a tomographic reconstructed model, and evaluates the simulated results in comparison to laboratory observations. As a simplified coarse-grained mathematical model, the current work provides a 3-D computational workflow to test various dynein-activation hypotheses, but it has a few limitations. For instance, only the mechanical influence of dynein arms is considered in the 3-D model. Other transverse connecting proteins, such as nexin, are embedded into the main body that provides continuity to the overall model. Linear elastic properties are assumed in the ciliary cytoplasm, and drag of the surrounding viscous fluid is neglected (in the study of the wild-type nodal cilia, the viscous drag is expected to be small [31]). These issues are limited by current observation and measurement techniques with for such small-scale transient structures during embryogenesis. When relevant information from biological research becomes available, more accurate computational analysis can be expected. Indeed, for mutant cilia with microtubule mislocalization, precise measurement and visualization of the abnormal axoneme is necessary; however, obtaining electronic tomographic datasets (with $1.6 \mathrm{~nm}$ slice-thickness) and videos of the movement of mutant embryonic cilia is extremely challenging. The current study provides a modelling and computational workflow for simulations of dynein-driven ciliary motion based on realistic model reconstruction of mutant nodal cilia. However, due to the limitations of the scanned samples, it focuses on one inv cilium. This study confirmed the rationality of modelling and investigating mutant ciliary movement with severe MT mislocalization. More importantly, it quantified the influence of doublet MT arrangement on ciliary movement and proposed the possible dynein-regulation pattern in inv nodal cilia. Based on this study with a 3-D reconstructed model established via tomographic data, future studies with a larger number of ciliary samples can be achieved by transmission microscopy to draw the generalized characteristics of ciliary moving mechanisms.

\section{Conclusion}

This work establishes a three-dimensional model of inv mutant nodal cilia based on electron tomography data that is able to realistically mimic the ultrastructure of the cilium. By applying the finite element method to simulate the dynein-driven ciliary movement, we convert this biological issue into a mechanical model to reveal the possible mechanism of the abnormal movement of the mutant cilia. The results confirm the validity of the distance- 


\section{Cellular Physiology Cell Physiol Biochem 2018;51:2843-2857 \begin{tabular}{l|l|l} 
and Biochemistry Published onlIne: 14 December 2018 & $\begin{array}{l}\text { ○ 2018 The Author(s). Published by S. Karger AG, Basel } \\
\text { www.karger.com/cpb }\end{array}$ \\
\hline
\end{tabular} \\ Yu et al.: Motility of Inv Mutant Nodal Cilia}

controlled dynein activation hypothesis. After analyzing the doublet MT morphology (angle and distance), we propose that, in the inv mutant, the mislocalization of the doublet MTs affects the angle of the doublet MTs as well as the interdoublet distance during ciliary motion, and thus induces the abnormality in ciliary movement. These conclusions will be more decisive when more samples can be obtained.

\section{Acknowledgements}

This study is supported by the National Natural Science Foundation of China (81471752) and the Beijing Scientific and Technology Program (Z181100003118007).

\section{Disclosure Statement}

The authors have no conflicts of interest to declare.

\section{References}

1 Eggenschwiler JT, Anderson KV: Cilia and developmental signaling. Annu Rev Cell Dev Biol 2007;23:345373.

2 Singla V, Reiter JF: The primary cilium as the cell's antenna: Signaling at a sensory organelle. Science 2006;313:629-633.

3 Sharma N, Berbari NF, Yoder BK: Ciliary dysfunction in developmental abnormalities and diseases. Curr Top Dev Biol 2008;85:371-427.

-4 Komatsu Y, Mishina Y: Establishment of left-right asymmetry in vertebrate development: the node in mouse embryos. Cell Mol Life Sci 2013;70:4659-4666.

-5 Essner JJ, Vogan KJ, Wagner MK, Tabin CJ, Yost HJ, Brueckner M: Conserved function for embryonic nodal cilia. Nature 2002;418:37-38.

-6 Levin M: Left-right asymmetry in embryonic development: a comprehensive review. Mech Dev 2005;122:325 .

7 Shinohara K, Hamada H: Cilia in left-right symmetry breaking. Cold Spring Harb Perspect Biol 2017;9:a028282.

8 Shinohara K, Kawasumi A, Takamatsu A, Yoshiba S, Botilde Y, Motoyama N, Reith W, Durand B, Shiratori H, Hamada H: Two rotating cilia in the node cavity are sufficient to break left-right symmetry in the mouse embryo. Nat Commun 2012;3:622.

-9 Hamada H: Roles of motile and immotile cilia in left-right symmetry breaking; in Nakanishi T, Markwald RR, Baldwin HS, Keller BB, Srivastava D, Yamagishi H (eds): Etiology and morphogenesis of congenital heart disease: from gene function and cellular interaction to morphology. Tokyo, Springer Japan, 2016, pp 57-65.

10 Song H, Hu J, Chen W, Elliott G, Andre P, Gao B, Yang Y: Planar cell polarity breaks the bilateral symmetry by controlling ciliary positioning. Nature 2010;466:378-382.

-11 Okada Y, Nonaka S, Tanaka Y, Saijoh Y, Hamada H, Hirokawa N: Abnormal nodal flow precedes situs inversus in iv and inv mice. Mol Cell 1999;4:459-468.

12 Lindemann CB, Lesich KA: The geometric clutch at 20: stripping gears or gaining traction? Reproduction 2015;150:R45-R53.

13 Lindemann CB: Experimental evidence for the geometric clutch hypothesis. Curr Top Dev Biol 2011;95:131.

14 Bayly PV, Wilson KS: Equations of interdoublet separation during flagella motion reveal mechanisms of wave propagation and instability. Biophys J 2014;107:1756-1772.

15 Satir P, Matsuoka T: Splitting the ciliary axoneme: implications for a "switch-point" model of dynein arm activity in ciliary motion. Cell Motil Cytoskeleton 1989;14:345-358. 


\section{Cellular Physiology Cell Physiol Biochem 2018;51:2843-2857 \begin{tabular}{ll|l} 
and Biochemistry Published onlIne: 14 December 2018 & $\begin{array}{l}\text { C } 2018 \text { The Author(s). Published by S. Karger AG, Basel } \\
\text { www.karger.com/cpb }\end{array}$ \\
\cline { 2 - 3 }
\end{tabular} \\ Yu et al.: Motility of Inv Mutant Nodal Cilia}

16 Brokaw CJ: Computer simulation of flagellar movement VIII: Coordination of dynein by local curvature control can generate helical bending waves. Cell Motil Cytoskeleton 2002;53:103-124.

17 Hilfinger A, Jülicher F: The chirality of ciliary beats. Phys Biol 2008;5:016003.

18 Brokaw CJ: Computer simulation of flagellar movement IX. Oscillation and symmetry breaking in a model for short flagella and nodal cilia. Cell Motil Cytoskeleton 2005;60:35-47.

-19 Shingyoji C, Higuchi H, Yoshimura M, Katayama E, Yanagida T: Dynein arms are oscillating force generators. Nature 1998;393:711-714.

20 Lindemann CB: The geometric clutch as a working hypothesis for future research on cilia and flagella. Ann N Y Acad Sci 2007;1101:477-493.

-21 Yan T, Wang W, Yang L, Chen K, Chen R, Han Y: Rich club disturbances of the human connectome from subjective cognitive decline to Alzheimer's disease. Theranostics 2018;8:3237-3255.

-22 Cartwright JHE, Piro 0, Tuval I: Fluid-dynamical basis of the embryonic development of left-right asymmetry in vertebrates. Proc Natl Acad Sci USA 2004;101:7234-7239.

23 Chen D, Norris D, Ventikos Y: The active and passive ciliary motion in the embryo node: a computational fluid dynamics model. J Biomech 2009;42:210-216.

24 Hirokawa N, Tanaka Y, Okada Y, Takeda S: Nodal flow and the generation of left-right asymmetry. Cell 2006;125:33-45.

25 Okada Y, Takeda S, Tanaka Y, Izpisúa Belmonte JC, Hirokawa N: Mechanism of nodal flow: a conserved symmetry breaking event in left-right axis determination. Cell 2005;121:633-644.

26 Smith DJ, Smith AA, Blake JR: Mathematical embryology: the fluid mechanics of nodal cilia. J Eng Math 2011;70:255-279.

27 Yan T, Feng Y, Liu T, Wang L, Mu N, Dong X, Liu Z, Qin T, Tang X, Zhao L: Theta Oscillations Related to Orientation Recognition in Unattended Condition: A vMMN Study. Front Behav Neurosci 2017;11:166.

-28 Smith DJ, Gaffney EA, Blake JR: Mathematical modelling of cilia-driven transport of biological fluids. Proc R Soc Lond A: Math Phys Eng Sci 2009;465:2417-2439.

-29 Mochizuki T, Saijoh Y, Tsuchiya K, Shirayoshi Y, Takai S, Taya C, Yonekawa H, Yamada K, Nihei H, Nakatsuji N, Overbeek PA, Hamada H, Yokoyama T: Cloning of inv, a gene that controls left/right asymmetry and kidney development. Nature 1998;395:177-181.

-30 Otto EA, Schermer B, Obara T, O’Toole JF, Hiller KS, Mueller AM, Ruf RG, Hoefele J, Beekmann F, Landau D, Foreman JW, Goodship JA, Strachan T, Kispert A, Wolf MT, Gagnadoux MF, Nivet H, Antignac C, Walz G, Drummond IA, Benzing T, Hildebrandt F: Mutations in INVS encoding inversin cause nephronophthisis type 2 , linking renal cystic disease to the function of primary cilia and left-right axis determination. Nat Genet 2003;34:413-420.

-31 Chen D, Zhong Y: A computational model of dynein activation patterns that can explain nodal cilia rotation. Biophys J 2015;109:35-48.

32 Shinohara K, Chen D, Nishida T, Misaki K, Yonemura S, Hamada H: Absence of radial spokes in mouse node cilia is required for rotational movement but confers ultrastructural instability as a trade-off. Dev Cell 2015;35:236-246.

-33 Kremer JR, Mastronarde DN, Mcintosh JR: Computer visualization of three-dimensional image data using IMOD. J Struct Biol 1996;116:71-76.

-34 Baba SA: Flexural rigidity and elastic constant of cilia. J Exp Biol 1972;56:459-467.

-35 Venier P, Maggs AC, Carlier MF, Pantaloni D: Analysis of microtubule rigidity using hydrodynamic flow and thermal fluctuations. J Biol Chem 1994;269:13353-13360.

-36 Kikumoto M, Kurachi M, Tosa V, Tashiro H: Flexural rigidity of individual microtubules measured by a buckling force with optical traps. Biophys J 2006;90:1687-1696.

37 Shingyoji C, Nakano I, Inoue Y, Higuchi H: Dynein arms are strain-dependent direction-switching force generators. Cytoskeleton 2015;72:388-401.

-38 Schmitz KA, Holcombwygle DL, Oberski DJ, Lindemann CB: Measurement of the force produced by an intact bull sperm flagellum in isometric arrest and estimation of the dynein stall force. Biophys J 2000;79:468478.

-39 Teff Z, Priel Z, Gheber LA: Forces applied by cilia measured on explants from mucociliary tissue. Biophys J 2007;92:1813-1823.

40 Minoura I, Yagi T, Kamiya R: Direct measurement of inter-doublet elasticity in flagellar axonemes. Cell Struct Funct 2000;24:27-33. 


\section{Cellular Physiology Cell Physiol Biochem 2018;51:2843-2857

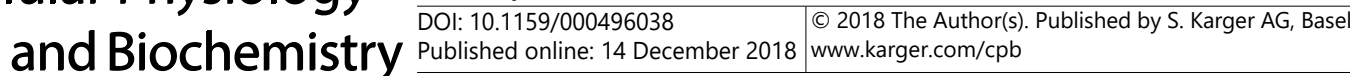 \\ Yu et al.: Motility of Inv Mutant Nodal Cilia}

41 Stein K, Tezduyar TE, Benney R: Automatic mesh update with the solid-extension mesh moving technique. Comput Meth Appl M 2004;193:2019-2032.

42 Cartwright JHE, Piro N, Piro 0, Tuval I: Embryonic nodal flow and the dynamics of nodal vesicular parcels. J R Soc Interface 2007;4:49-55.

43 Okada Y, Nonaka S, Tanaka Y, Saijoh Y, Hamada H, Hirokawa N: Abnormal nodal flow precedes situs inversus in iv and inv mice. Mol Cell 1999;4:459-468.

44 Yokoyama T, Copeland N, Jenkins N, Montgomery C, Elder F, Overbeek P: Reversal of left-right asymmetry: a situs inversus mutation. Science 1993;260:679-682.

-45 Chen D, Zhong Y, Shinohara K, Nishida T, Hasegawa T, Hamada H: The dynein-triggered ciliary motion in embryonic nodes: An exploratory study based on computational models. Biomed Mater Eng 2014;24:24952501.

46 Tsuji T, Matsuo K, Nakahari T, Marunaka Y, Yokoyama T: Structural basis of the Inv compartment and ciliary abnormalities in Inv/nphp2 mutant mice. Cytoskeleton 2016;73:45-56.

47 Lindemann CB: Testing the geometric clutch hypothesis. Biol Cell 2004;96:681-690. 